\title{
Delineating Amyloid Plaque Associated Neuronal Sphingolipids in Transgenic Alzheimer's Disease Mice (tgArcSwe) Using MALDI Imaging Mass Spectrometry
}

\author{
Ibrahim Kaya, ${ }^{\dagger, \nabla}$ Dimitri Brinet, ${ }^{\dagger \dagger}, \nabla$ Wojciech Michno, ${ }^{\dagger}$ Stina Syvänen, ${ }^{\S}$ Dag Sehlin, ${ }^{\S}$ \\ Henrik Zetterberg, ${ }^{\dagger, \|, \perp}$ Kaj Blennow, ${ }^{\dagger, \|}$ and Jörg Hanrieder ${ }^{* \dagger, \perp, \# \mathbb{C}}$
}

${ }^{\dagger}$ Department of Psychiatry and Neurochemistry, Sahlgrenska Academy at the University of Gothenburg, 43180 Mölndal, Sweden

${ }^{\ddagger}$ Department of Chemistry and Molecular Biology, University of Gothenburg, 41296 Gothenburg, Sweden

${ }^{\S}$ Department of Public Health and Caring Sciences, Uppsala University, 75237 Uppsala, Sweden

"Clinical Neurochemistry Laboratory, Sahlgrenska University Hospital, 43180 Mölndal, Sweden

${ }^{\perp}$ Department of Molecular Neuroscience, UCL Institute of Neurology, University College London, London WC1N 3BG, United Kingdom

${ }^{\#}$ Department of Chemistry and Chemical Engineering, Chalmers University of Technology, 41296 Gothenburg, Sweden

\section{Supporting Information}

\begin{abstract}
The major pathological hallmarks of Alzheimer's disease $(\mathrm{AD})$ are the progressive aggregation and accumulation of beta-amyloid $(\mathrm{A} \beta)$ and hyperphosphorylated tau protein into neurotoxic deposits. A $\beta$ aggregation has been suggested as the critical early inducer, driving the disease progression. However, the factors that promote neurotoxic $\mathrm{A} \beta$ aggregation remain elusive. Imaging mass spectrometry (IMS) is a powerful technique to comprehensively elucidate the spatial distribution patterns of lipids, peptides, and proteins in biological tissue sections. In the present study, matrix-assisted laser desorption/ionization (MALDI) mass spectrometry (MS)-based imaging was used on transgenic Alzheimer's disease mouse (tgArcSwe) brain tissue to investigate the sphingolipid microenvironment of individual A $\beta$ plaques and elucidate plaque-associated sphingolipid alterations. Multivariate data analysis was used to interrogate the IMS data for identifying pathologically relevant, anatomical features based on their lipid chemical profile. This approach revealed sphingolipid species that distinctly located to cortical and hippocampal deposits, whose $\mathrm{A} \beta$ identity was further verified using fluorescent amyloid staining and immunohistochemistry. Subsequent multivariate statistical analysis of the spectral data revealed significant localization of gangliosides and ceramides species to $\mathrm{A} \beta$ positive plaques, which was accompanied by distinct local reduction of sulfatides. These plaque-associated changes in sphingolipid levels implicate a functional role of sphingolipid metabolism in $\mathrm{A} \beta$ plaque pathology and $\mathrm{AD}$ pathogenesis. Taken together, the presented data highlight the potential of imaging mass spectrometry as a powerful approach for probing $\mathrm{A} \beta$ plaque-associated lipid changes underlying $\mathrm{AD}$ pathology.
\end{abstract}

KEYWORDS: Alzheimer's disease, amyloid- $\beta$ plaque pathology, MALDI imaging mass spectrometry, sphingolipids, tgArcSwe

\section{INTRODUCTION}

Alzheimer's disease $(\mathrm{AD})$ is the most prevalent neurodegenerative disorder. The neuropathology of $\mathrm{AD}$ is characterized by the formation of protein deposits in the brain including intercellular neurofibrillary tangles consisting of hyperphosphorylated tau protein ${ }^{1}$ and extracellular plaques formed by aggregated amyloid- $\beta(\mathrm{A} \beta)$ peptides. ${ }^{2,3} \mathrm{~A} \beta$ peptides rapidly aggregate to oligomers, protofibrils and fibrils, eventually leading to formation of extracellular plaques. Little is known about the molecular mechanisms of how monomeric $\mathrm{A} \beta$ peptides are converted to neurotoxic, aggregated forms that are rich in $\beta$-sheet motifs. A number of biochemical and clinical studies suggest that in addition to peptide centric mechanisms, changes in neuronal lipid metabolism may be implicated in $\mathrm{AD}$ pathogenesis and plaque pathology in particular. ${ }^{4,5}$ Further- more, genetic predisposition with the apolipoprotein $\mathrm{E}(A P O E)$ $\varepsilon 4$ allele, a lipid transporter protein, was identified as the major risk factor to develop sporadic $\mathrm{AD}$, further suggesting a possible role for lipids in $\mathrm{AD}$ pathogenesis. ${ }^{6-9}$ Hence, several studies on the relevance of neuronal lipid species associated with $\mathrm{AD}$ pathology, including sphingolipids, ${ }^{10,11}$ cholesterol, $^{12}$ and phospholipids ${ }^{13}$ have been reported. In particular, a potential role in $\mathrm{AD}$ pathogenesis has been suggested for a number of sphingolipid species, including ceramides, ${ }^{7,14}$ sulfatides, ${ }^{14,15}$ and

Special Issue: Monitoring Molecules in Neuroscience 2016

Received: November 14, 2016

Accepted: December 16, 2016

Published: December 16, 2016 


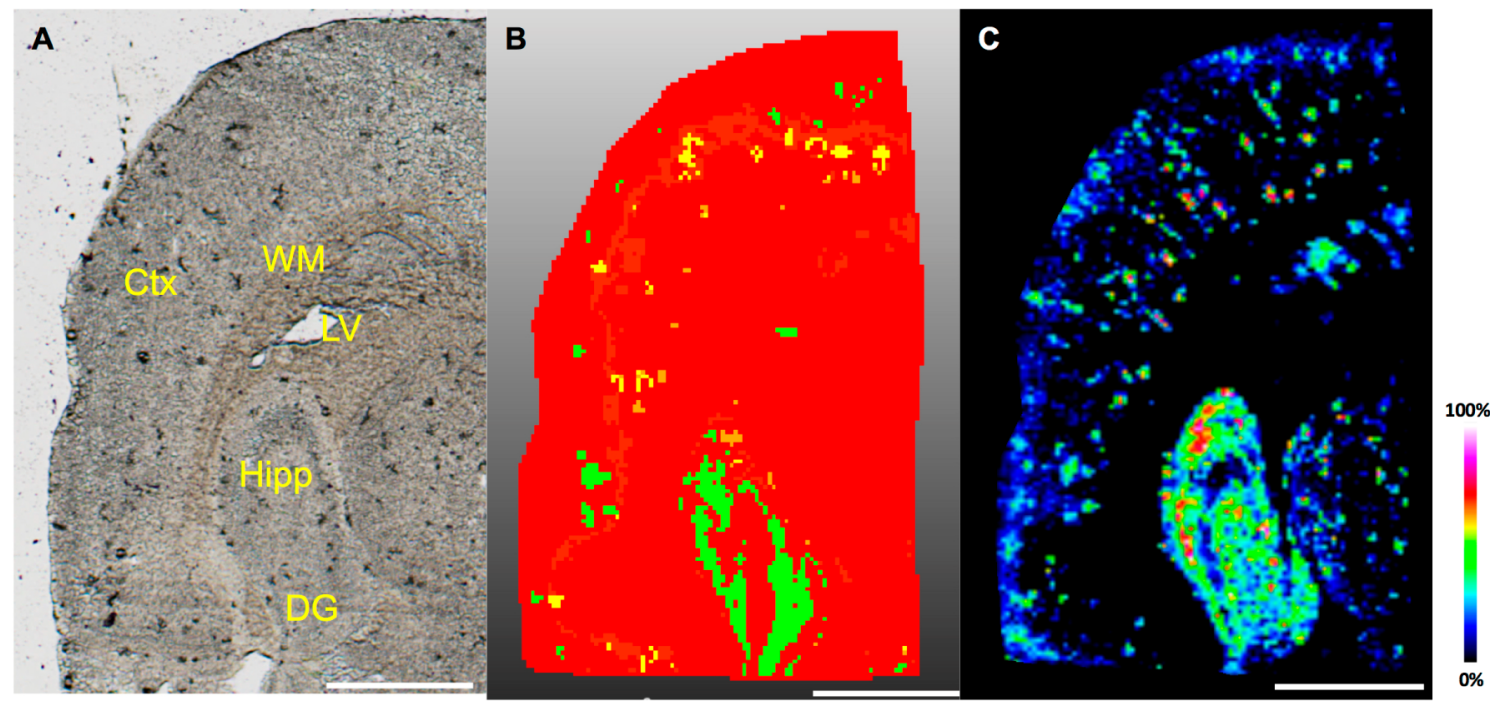

Figure 1. Multivariate image analysis of MALDI-IMS data reveals cortical and hippocampal sphingolipid accumulations in tgArcSwe mouse brain. (A) Bright field image showing dorsolateral part of a coronal section used for IMS analysis. Anatomical regions are annotated as follows: Ctx, cortex; WM, white matter; LV, lateral ventricle; hipp, hippocampus; DG, dentate gyrus of the hippocampus. (B) Segmentation map obtained from multivariate image analysis of the MALDI-IMS data. Bisecting k-means based hierarchical clustering analysis identified plaque-like features (green, yellow, orange) in the cortex and hippocampus based on the encoded chemical information. Inspection of the associated variables $(m / z$ values) expressing the clustering behavior, revealed $m / z$ values localizing to anatomical features. (C) This includes, e.g., Cer(d18:1/12:0) at $m / z$ 480.5 as further verified by the corresponding single ion image as a representative plaque associate sphingolipid species. Scale bar $=0.5 \mathrm{~mm}$.

gangliosides. $^{16-19}$ Therefore, in order to further delineate the relevance of sphingolipids in $\mathrm{AD}$ pathogenesis, it is important to outline their spatial distribution profile in situ. Genetically altered mouse models are of central relevance to probe the molecular mechanisms of $\mathrm{AD}$ pathology. ${ }^{20,21}$ For instance, a transgenic mouse model of $\mathrm{AD}$, with mice carrying both the Arctic (E693G) and the Swedish (K670M/N671L) mutations ( $\operatorname{tgArcSwe}$ ) of amyloid precursor protein (APP) has been developed. These mice exhibit in extensive $\mathrm{A} \beta$ deposition with an onset at the age of 5-6 months, and are a well-suited model system to study molecular mechanisms for $\mathrm{A} \beta$ deposition. ${ }^{22}$

In order to characterize disease pathology in situ, advanced molecular imaging techniques are required including imaging mass spectrometry (IMS). This technique is often referred as molecular histology and allows to generate spatial intensity distribution maps of molecular species in complex biological tissues. $^{23}$ In particular, matrix assisted laser desorption/ ionization (MALDI) based IMS is a well suited IMS modality to probe neuronal lipids, ${ }^{24}$ including gangliosides ${ }^{25}$ as well as for in situ characterization of endogenous neuropeptides ${ }^{26,27}$ associated with neurodegenerative disease pathology. Indeed, MALDI-IMS, has been previously successfully demonstrated for probing $\mathrm{A} \beta$ peptide pathology in tgArcSwe mice. ${ }^{28}$

In the present study, MALDI-IMS was employed to examine the sphingolipid microenvironment, including ganglioside-, sulfatide- and ceramide- localizations to cortical and hippocampal $\mathrm{A} \beta$ plaques in tgArcSwe mice. Here, IMS was used in conjunction with multivariate statistical analysis tools to identify regional plaque-associated changes in neuronal sphingolipid chemistry.

\section{RESULTS AND DISCUSSION}

MALDI Imaging MS Reveals Distinct Localization of Neuronal Sphingolipid Species on the Cortical and Hippocampal Amyloid- $\boldsymbol{\beta}$ Plaques. In MALDI-IMS, sample preparation includes deposition of a matrix species, dissolved in an aqueous/organic solvent system onto the tissue sections. However, this process can result in lateral analyte diffusion and disturbance of tissue morphology. In contrast, sublimation is a solvent free approach for matrix deposition that overcomes analyte delocalization issues arising with wet matrix application and hence allows for high-spatial resolution. ${ }^{29}$ Previously, sublimation of 1,5-diaminonaphthalene (1,5-DAN) for MALDI IMS was shown to give enhanced signal intensity for neuronal lipid species at high spatial resolution, particularly in negative ion mode. ${ }^{30}$ In the present study, 1,5-DAN sublimation was therefore used for MALDI-IMS analysis of neuronal sphingolipids in tgArcSwe mice. The acquired image data were investigated by using unsupervised multivariate statistics based on hierarchical clustering analysis (bisecting k-means) in order to obtain image segmentation of anatomical regions of interest based on their lipid chemical profile. Here, image segmentation identified deposit-like features in the cortex and hippocampus (Figure 1A,B). These features were assigned as individual regions of interest (ROI) and correlated to the processed MS data, in order to identify the associated chemical species that allowed image segmentation of the regions.

Inspection of the corresponding variables revealed localization of various sphingolipid species to the plaque-like features, including ceramides and gangliosides. Visualization of single ion intensity distributions (i.e., single ion images) for the individual ceramide (e.g., $\operatorname{Cer}(\mathrm{d} 18: 1 / 12: 0), m / z$ 480.5, Figure 1C) and ganglioside species (Figure 4) showed a consistent deposit-like distribution patterns throughout the cortical and hippocampal regions. Hence, the here employed workflow for unbiased segmentation of the complex imaging data was found to be a strong approach for elucidating the chemical composition of the plaque-like deposits.

Amyloid- $\boldsymbol{\beta}$ Plaque Identification and Validation by Subsequent Fluorescent Histochemical Amyloid Staining. In order to verify the potential plaque identity as observed for individual ceramide- and ganglioside-accumulations, includ- 


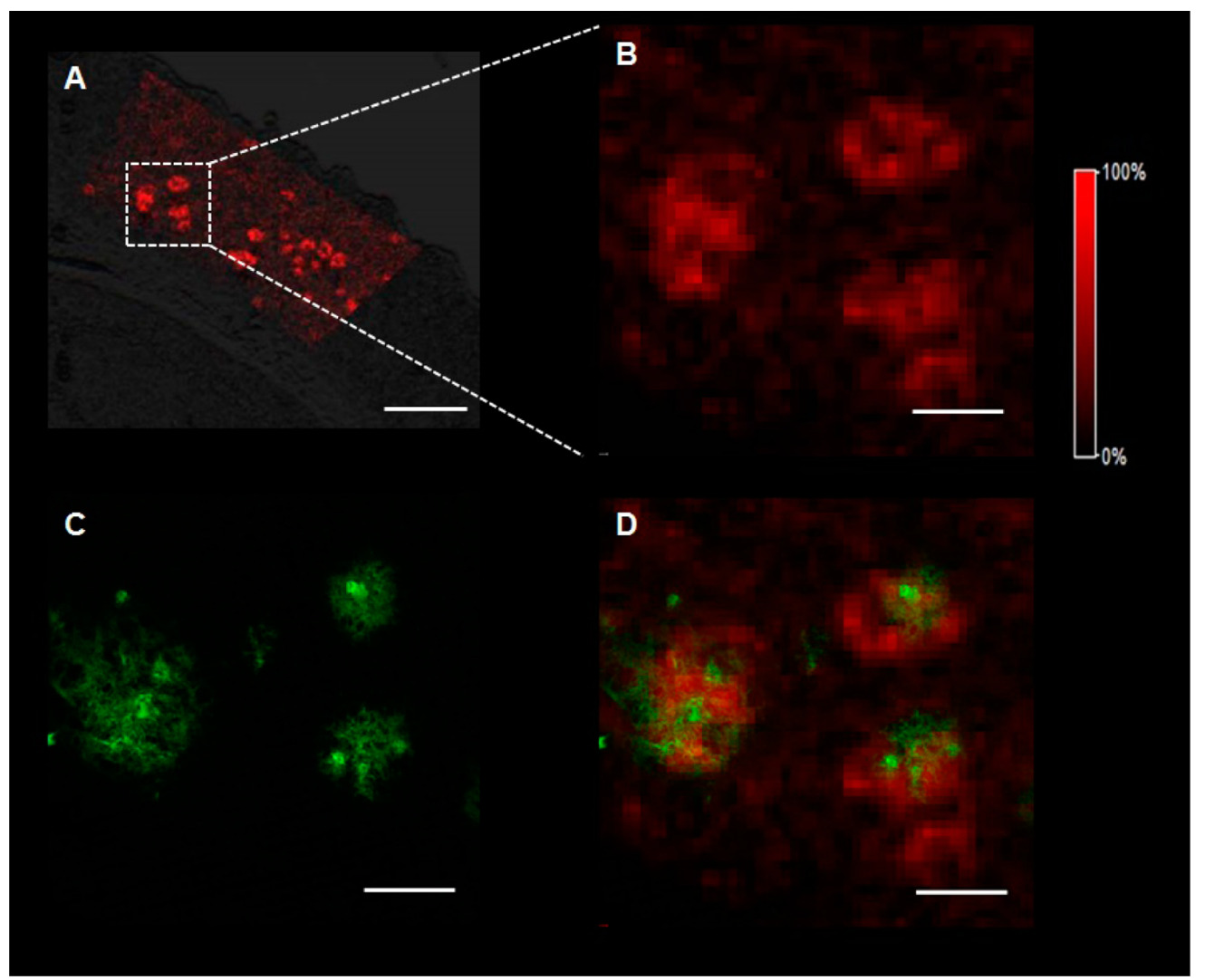

Figure 2. Identification of $\mathrm{A} \beta$ deposits localized with MALDI-IMS using fluorescent amyloid staining. (A) Single ion image of $m / z 480.5$ (Cer(d18:1/12:0)) using MALDI-IMS with $10 \mu \mathrm{m}$ spatial resolution. (B) Magnified single ion images of $\mathrm{m} / z$ 480.5 from the cortex. (C) Histologically identified plaque, stained with h-FTAA reveals A $\beta$ fibril structures in the cortical regions (D) colocalized with the accumulation observed in the magnified single ion images of $\mathrm{m} / z$ 480.5. Scale bars for $(\mathrm{A})=400 \mu \mathrm{m}$ and $(\mathrm{B}-\mathrm{D})=100 \mu \mathrm{m}$.

ing e.g. Cer(d18:1/12:0) (Figure 2A, B), histochemical staining was performed using a luminescent conjugated oligothiophene (LCO), h-FTAA, a fluorescent amyloid probe (Figure 2C). ${ }^{31}$ Here, a strong colocalization was observed for amyloid staining and distributions profiles of distinct sphingolipid species as identified by MALDI-IMS (Figure 2D). Furthermore, in order to confirm the specificity of the chemical amyloid staining to $\mathrm{A} \beta$ fibrils, double staining of h-FTAA and $\mathrm{A} \beta$ immunohistochemistry (IHC) was performed using a monoclonal $\mathrm{A} \beta$ antibody $(\mathrm{A} \beta 1-16,6 \mathrm{E} 10)$. The fluorescent imaging results show clear double positive staining of the plaques for both hFTAA and $\mathrm{A} \beta$, thereby confirming the $\mathrm{A} \beta$ identity of the hFTAA stained deposits (Supporting Information Figure S-1AC).

In addition, to further verify the lipid localizations to amyloid plaques, a bottom up multivariate, correlation analysis approach was used. Here, both imaging MS data and fluorescent staining data (h-FTAA and IHC) were coregistered in the SciLs software (Figure S-1D,E). This was followed by annotation of h-FTAA/A $\beta$ double positive features in the fluorescence imaging data and correlation analysis to the whole imaging data set. The results revealed the most prominent variables $(\mathrm{m} /$ $z$ values) that correlate with $\mathrm{A} \beta$ and h-FTAA double positive plaque regions (Figure $S-1 D, E$ ). Here, the most significant peaks above a certain threshold at $p<0.05$ significance level could indeed be attributed to sphingolipid species, including $\mathrm{m} /$ $z$ peaks corresponding to gangliosides GM2, GM3 as well as ceramide species $\operatorname{Cer}(\mathrm{d} 18: 1 / 12: 0), \operatorname{Cer}(\mathrm{d} 18: 1 / 14: 0)$ and
Cer(d18:1/18:0) and sulfatides, thereby further supporting the multivariate image analysis results (Figure S-1D,E).

Plaque-Associated Alterations of Sphingolipids. Neuronal membrane-derived sphingolipids play important roles in biological processes. This includes signal transduction, cell recognition and senescence, either through regulating the roles of membrane-associated receptors or by acting as a precursor of bioactive lipid mediators. Recently, it has been reported that deregulated sphingolipid metabolism could play a role in $\mathrm{AD}$ associated amyloid processing, $\mathrm{A} \beta$ fibrillogenesis and impairment of synaptic function. ${ }^{11,32}$ It has been widely reported that $\mathrm{A} \beta$ interacts with gangliosides ${ }^{17,33}$ with high affinities, ${ }^{34}$ thereby adopting an altered structural conformation through its binding to GM1 and potentially other ganglioside species. ${ }^{16}$ This results in ganglioside- $\mathrm{A} \beta$ peptide adduct formation, which in turn was found to trigger $\mathrm{A} \beta$ fibrillogenesis in $\mathrm{AD}$ brain. ${ }^{35,36}$ Moreover, increased membrane-associated oxidative stress and excessive production and accumulation of ceramides are observed along with sulfatide reduction in association with $\mathrm{AD} .^{37,38}$ In order to investigate the sphingolipid content for individual $\mathrm{A} \beta$ plaques in different brain regions as previously outlined by image analysis, MALDI-IMS spectral data of individual ROI, comprising $\mathrm{A} \beta$ deposits and corresponding adjacent control areas, were annotated and evaluated by statistical analysis. Here, IMS data from amyloid-positive deposit regions and adjacent control regions within either the cortex or the hippocampus were extracted from the data of three different animals $(n=3)$ and submitted for two class, paired t-statistics. Nonplaque control regions, representing aggregate-free areas were assigned 
based on the fluorescent amyloid staining. Statistical analysis was performed using the SAM (statistical analysis of microarrays) approach for unbiased interrogation of the imaging data sets.

In SAM, a score is calculated for each variable $(\mathrm{m} / \mathrm{z}$ value) that measures the strength of the relationship between peak intensity and the sample group (i.e., response variable; plaque vs control). Here, repeated permutations of the data were used in order to determine if the intensity of each variable was significantly different for the two sample groups. The results are presented in plots where the calculated (observed) score is displayed for each variable $(\mathrm{m} / \mathrm{z}$ value) as a function of the "expected" score, which is calculated from the fluctuations in the data, assuming that there is no difference between the groups. An observed score that is significantly larger than the expected score thus indicates a significant difference between the groups for this variable. The results show a significant $(p<$ 0.05 ) increase (Figure 3, red) or decrease (Figure 3, green) of various $m / z$ signals in all plaque ROIs compared to the control areas. A majority of the significantly different peaks could be

\section{A Cortex}
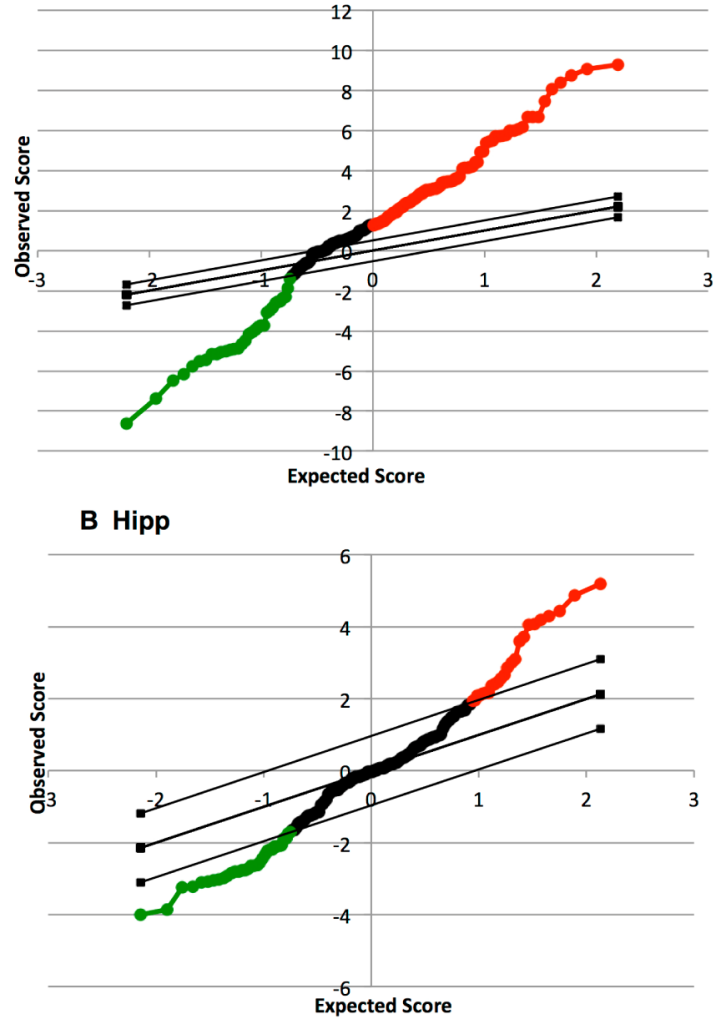

Figure 3. Statistical analysis of peak intensity differences between plaques and adjacent control areas. Two class, paired analysis was performed using the SAM technique in the somatosensory cortex (A) and hippocampus (B). The graphs depict for each variable (peak $\mathrm{m} / \mathrm{z}$ value) the observed SAM score, indicating the relative difference between control and plaque. The expected score indicates the random fluctuation when there is no difference between the two groups. Red marked variables indicate $m / z$ values that are increased in the plaque compared to the control, where the difference of the observed (SAM) versus the expected score is above a certain threshold (indicated by thin lines), representing the $95 \%$ significance level. Green variables indicate $m / z$ peaks that are lower in the plaque region as compared to the immediate local proximity. assigned to sphingolipid species. This included gangliosides and ceramides that were increased in hippocampal and cortical amyloid deposits (Figures $4 \mathrm{~A}-\mathrm{H}$ and S-2). In contrast, sulfatide species displayed an inverse localization pattern and were decreased in plaque ROI in both cortex and hippocampus (Figures $5 \mathrm{~A}-\mathrm{D}$ and $\mathrm{S}-2$ ). The distinct localization of ceramides and gangliosides to the plaque regions was prominent when inspecting the ROI spectral data (Figure S-3a-c). Here, characteristically higher peak intensities are observed for $\mathrm{m} / \mathrm{z}$ peaks corresponding to, e.g, Cer(d18:1/18:0), GM2(d18:1/ 18:0), and GM3(d18:1/18:0) (Figure S-3d). Moreover, to further demonstrate that the different peak levels are related to amyloid plaque pathology, MALDI IMS data from 18 month old control animals were acquired (Figures S-3a and S-4). Here, as expected no plaque pathology was observed. Furthermore, no plaque-like accumulation pattern were observed for the respective sphingolipid species or any other lipid peaks (Figure S-4). In addition to the general statistics of the ROI spectral data, the technical variation of the here employed sample preparation and acquisition was estimated. For that three sections from one animal were collected and prepared and analyzed at three different occasions. Following ROI annotation, spectra extraction, and processing, the average relative standard deviation of the three technical replicates was calculated and found to be $20.75 \%$.

In order to verify the lipid identities and draw biologically relevant conclusions, further means of molecular identification beyond accurate mass assignments are imperative. Therefore, MS/MS experiments were performed directly in situ on the tissue sections following DAN sublimation. Here, the majority of the significantly changed sphingolipid species were successfully identified based on their fragment ions (Figure S5). ${ }^{39,40}$

Based on the accurate mass and MS/MS data, the various $m$ / $z$ signals could be attributed to several ceramides, including $\mathrm{m} / z$ 564.6, $\operatorname{Cer}(\mathrm{d} 18: 1 / 18: 0), m / z$ 508.5, $\operatorname{Cer}(\mathrm{d} 18: 1 / 14: 0)$ and $m / z$ 480.5, $\operatorname{Cer}(\mathrm{d} 18: 1 / 12: 0$ ) (Figure $\mathrm{S}-5 \mathrm{a}-\mathrm{c}$ ) as well as ganglioside species such as $m / z$ 1544.9, GM1(d18:1/18:0), $m / z$ 1382.8, GM2(d18:1/18:0) and $m / z$ 1179.7, GM3(d18:1/18:0) (Figure S-5d-f).

Inspection of single ion images generated for the various sphingolipid species revealed that both gangliosides and ceramides were enriched in $\mathrm{A} \beta$ deposits in the hippocampus and somatosensory cortex (Figure 4A-H). In contrast, some cortical sulfatides (ST) detected with MALDI-IMS and validated by in situ MS/MS, including ST(18:0) (( $3^{\prime}$ sulfo)Gal $\beta$-Cer(d18:1/18:0)) and ST(24:1) (( $3^{\prime}$-sulfo $) \mathrm{Gal} \beta$ Cer(d18:1/24:1)) (Figure S-5g,h), were significantly decreased in the plaques as compared to the adjacent gray matter control area (Figure 5A-D).

Previous studies reported that gangliosides GM2, GM3 increased in multiple brain regions including frontal and temporal cortex whereas GM1 was decreased in $\mathrm{AD}$ compared with matched controls. ${ }^{11,41,42}$ In the present study, no substantial changes in plaque-associated GM1 levels were observed in either of the brain regions. This is deviating from previous observations, where a reduction in gangliosides was found in gray matter regions in $\mathrm{AD}$ patients ${ }^{43}$ together with a corresponding increase in CSF levels of GM1. ${ }^{18}$ This can be explained twofold. First, previous studies relied solely on antibody based methods for characterizing ganglioside expression in situ. Mass spectrometry is superior with respect to molecular specificity and allows thereby accurate annotation 

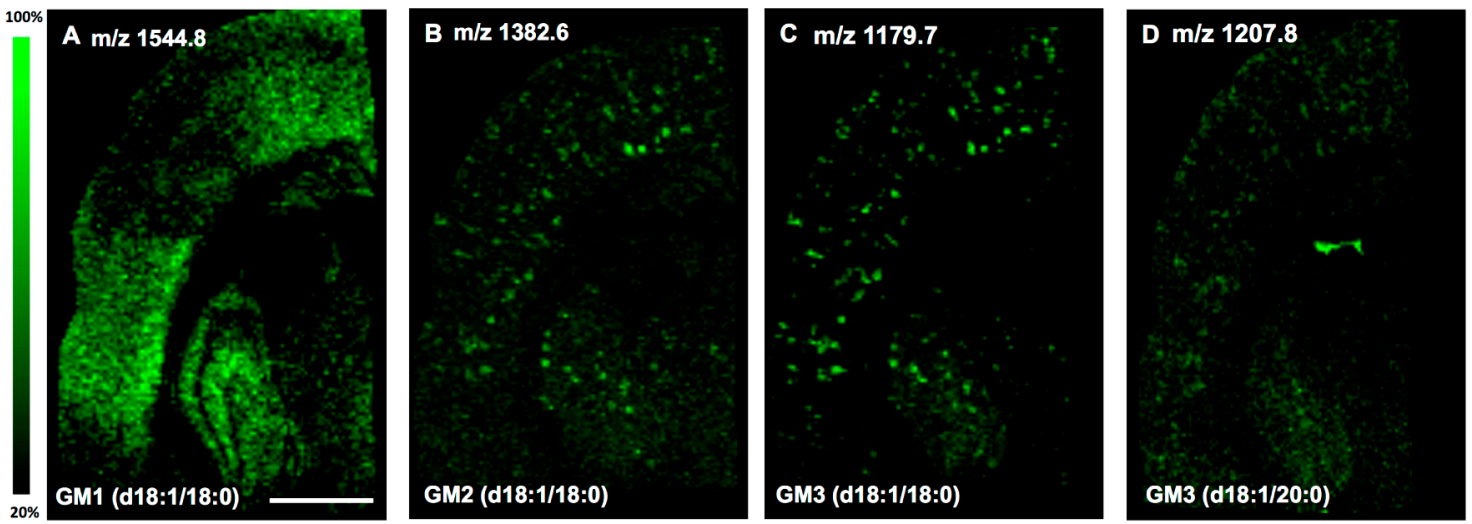

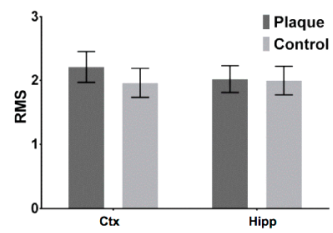

GM1 (d18:1/18:0)

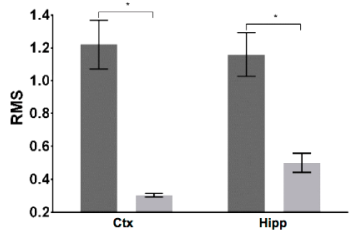

GM2 (d18:1/18:0)

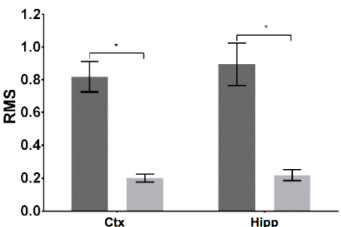

GM3 (d18:1/18:0)

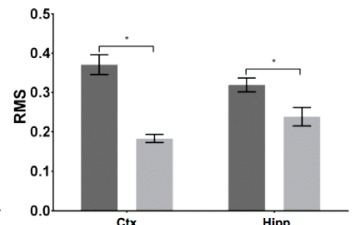

GM3 (d18:1/20:0)
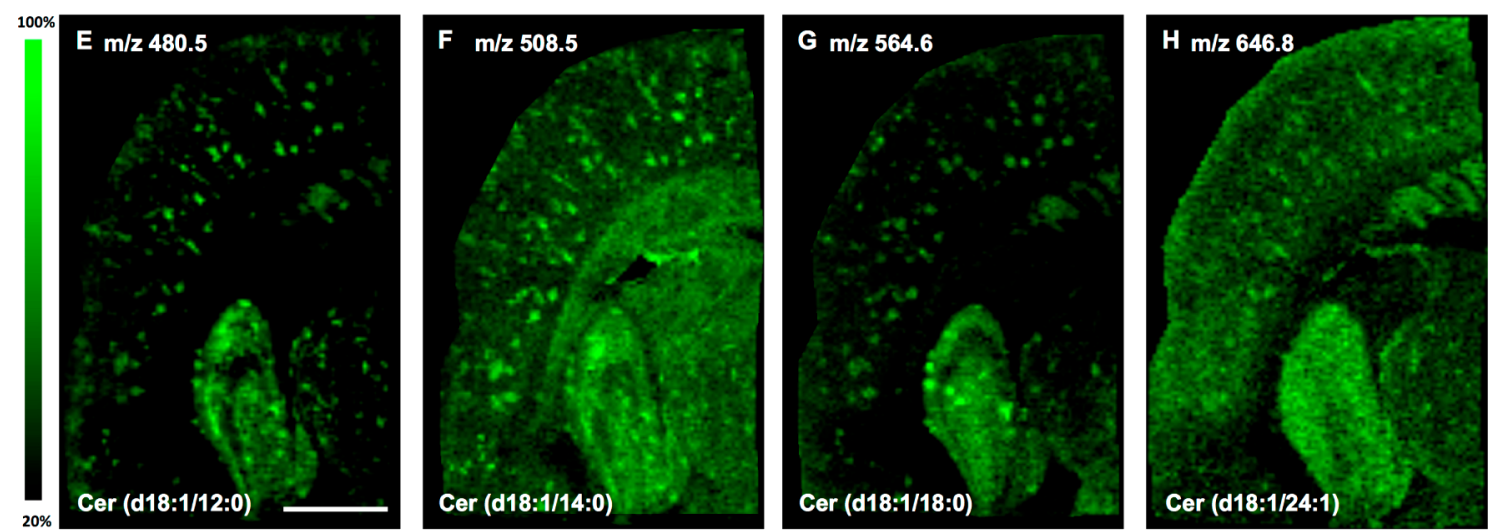

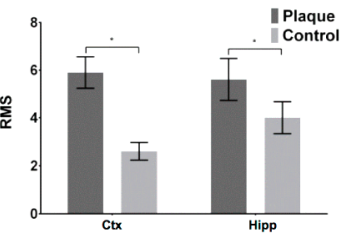

Cer(d18:1/12:0)

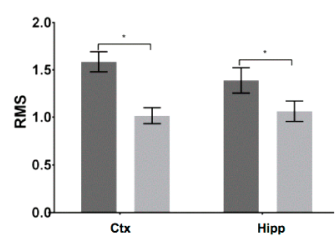

Cer(d18:1/14:0)

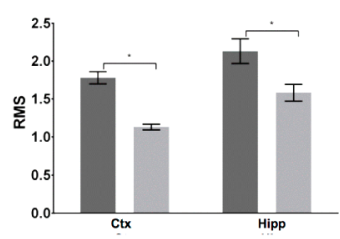

Cer(d18:1/18:0)

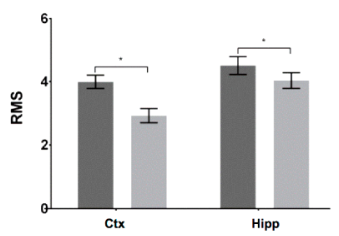

Cer(d18:1/24:1)

Figure 4. Cortical and hippocampal plaque-associated accumulation of gangliosides and ceramides in tgArcSwe brain. Statistical analysis (SAM) of ROI spectral data revealed plaque-associated increase of gangliosides and ceramide species in the cortex (Ctx) and hippocampus (Hipp). Individual plaque ROIs were compared with adjacent control regions within the same tissue section and brain region. (A-D) Single ion images demonstrated that GM1(d18:1/16:0), $[\mathrm{M}-\mathrm{H}]^{-} 1544.9$ (A) was prominent in both brain regions but did not localize to plaques. In contrast, GM2(d18:1/18:0), $[\mathrm{M}-\mathrm{H}]^{-} 1382.8(\mathrm{~B}), \mathrm{GM} 3(\mathrm{~d} 18: 1 / 18: 0),[\mathrm{M}-\mathrm{H}]^{-} 1179.7(\mathrm{C})$, and GM3(d18:1/20:0), $[\mathrm{M}-\mathrm{H}]^{-} 1207.8(\mathrm{D})$ were found to be significantly $(* p$ $<0.05$ ) increased in both cortical and hippocampal plaques. (E-H) Several ceramides, including Cer(d18:1/12:0), $[\mathrm{M}-\mathrm{H}]^{-} 480.5(\mathrm{E})$; $\operatorname{Cer}(\mathrm{d} 18: 1 / 14: 0),[\mathrm{M}-\mathrm{H}]^{-} 508.5(\mathrm{~F})$; Cer (d18:1/18:0), $[\mathrm{M}-\mathrm{H}]^{-} 564.6(\mathrm{G})$, and $\operatorname{Cer}(\mathrm{d} 18: 1 / 24: 1),[\mathrm{M}-\mathrm{H}]^{-} 646.8(\mathrm{H})$ were found to be significantly $(* p<0.05)$ elevated in the plaques as compared to the control areas in both regions (mean $\pm \mathrm{SD})$. Scale bars for $(\mathrm{A})-(\mathrm{H})=0.5 \mathrm{~mm}$.

and characterization of local changes of distinct ganglioside isoforms. Second, the here reported localizations were spatially resolved and attributed to plaque features at $10-30 \mu \mathrm{m}$ spatial resolution. Previous findings were performed on tissue extracts where spatially confined changes are convoluted.

Interestingly, an accumulation of ceramides was observed in the vicinity of the plaque regions where sulfatides were decreased (Figure 5E, F). Ceramide increase and sulfatide reduction $^{14,38}$ can be explained by accelerated lysosomal degradation of neuronal gangliosides (GM1, GD1, and GT1) and sulfatides in the plaque regions, as observed during cell death in $\mathrm{AD}$ pathology. ${ }^{42,44}$ This can furthermore trigger an excessive accumulation of ceramides and ganglioside (GM2, GM3) species around cortical and hippocampal $\mathrm{A} \beta$ plaques either as a consequence of altered ganglioside catabolism or inhibited lysosomal storage and degradation of GM2 and 

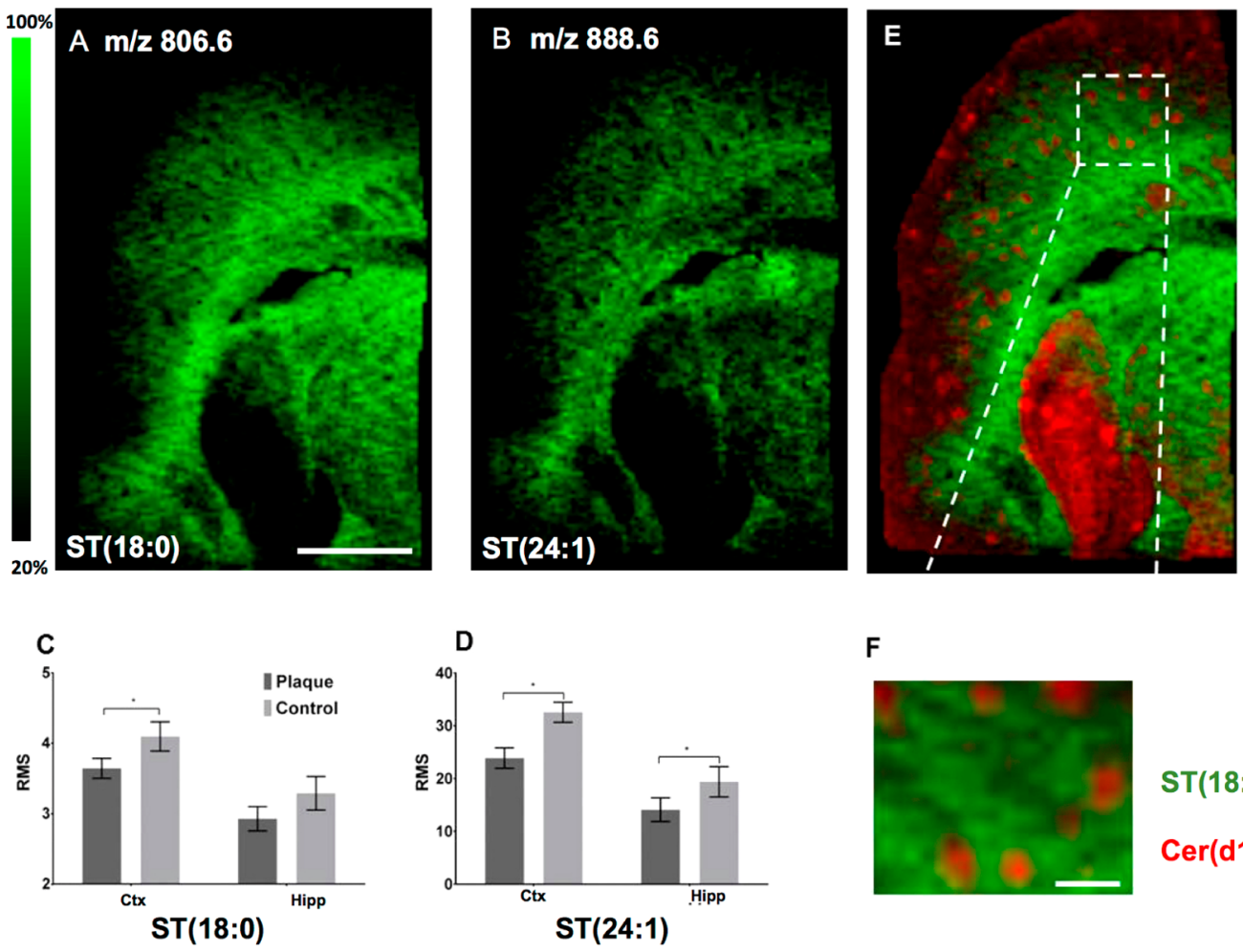

$\mathbf{F}$

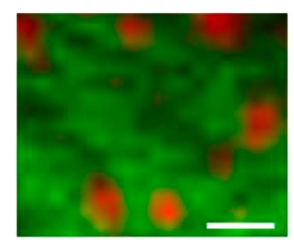

ST(18:0)

Cer(d18:1/18:0)

Figure 5. Plaque associated reduction of sulfatides. Statistical analysis ROI spectral data revealed decreased sulfatide signals in plaque regions within the cortex (Ctx) and hippocampus (Hipp). (A-B) Sulfatide species, including ST(18:0) ( $3^{\prime}$-sulfo)Gal $\beta$-Cer(d18:1/18:0), $m / z$ 806.6 (A, C); and $\mathrm{ST}(24: 1)\left(\left(3^{\prime}\right.\right.$-sulfo $) \mathrm{Gal} \beta$-Cer(d18:1/24:1)), $m / z$ 888.6 (B, D), were found to be significantly decreased in the deposits as compared to the control areas in both Ctx and Hipp $(p<0.05$; mean \pm SD) as verified by the single ion images $(\mathrm{E}, \mathrm{F})$. An overlay of single ion images of ST(18:0), $m / z$ 806.6 (green), with the corresponding ceramide residue (Cer(d18:1/18:0), $m / z 564.6$ (red)), highlights complementary localization of both species. (F) Magnification of marked inset (E) in the lateral Ctx. Scale bars for (A), (B), and (E) $=0.5 \mathrm{~mm}, F=0.2 \mathrm{~mm}$.

GM3. ${ }^{44}$ Similarly, an accumulation of cortical GM2 and GM3 levels and characteristic distribution to extracellular deposits was recently reported in a genetic mouse model of Hunter's disease, a lysosomal storage disorder. ${ }^{45}$ These and our findings suggest that impaired ganglioside hydrolases and lysosomal degradation might be associated with $\mathrm{AD}$ plaque pathology. This is presumably as a consequence of amyloid aggregation induced neuronal dysfunction that leads to pathological characteristics of lysosomal storage disease, and gangliosidosis, respectively. Taken together, the data suggest a significant role of altered $\mathrm{A} \beta$ plaque-associated sphingolipid metabolism in transgenic $\mathrm{AD}$ mice (tgArcSwe).

\section{CONCLUSION}

The present study demonstrates the potential of MALDI-IMS for in situ interrogation of the sphingolipid content in individual $\mathrm{A} \beta$ plaques. The results revealed significant plaqueassociated accumulation of distinct ceramide and ganglioside species along with sulfatide depletion, suggesting a prominent role of sphingolipid metabolism in $\mathrm{AD}$ pathology. These data highlight the potential of this technique for in situ probing of plaque-associated lipid chemical changes in neurodegenerative diseases. Further studies are warranted to examine how this translates to patients with $\mathrm{AD}$.

\section{METHODS}

Chemicals and Reagents. All chemicals for matrix and solvent preparation were pro-analysis grade and obtained from Sigma-Aldrich (St. Louis, MO), unless otherwise specified. TissueTek optimal cutting temperature (OCT) compound was purchased from Sakura Finetek
(AJ Alphen aan den Rijn, The Netherlands). The $\mathrm{ddH}_{2} \mathrm{O}$ was obtained from a Milli-Q purification system (Millipore Corporation, Merck Millipore, Billerica, MA).

Animals. Transgenic mice ( $n=3 ; 2$ male, 1 female), 18 months of age, with the Arctic (E693G) and Swedish (K670N, M671L) mutations ( $\operatorname{tgArcSwe}$ ) of human APP were reared ad libitum at the animal facility at Uppsala University under a $12 \mathrm{~h} / 12 \mathrm{~h}$ light cycle. ${ }^{22}$ The animals were anesthetized with isoflurane and sacrificed by decapitation. The brains were dissected quickly with $3 \mathrm{~min}$ postmortem delay and frozen on dry ice. All animal procedures were approved by an ethical committee and performed in compliance with national and local animal care and use guidelines ( $\mathrm{DNr} \# \mathrm{C} 17 / 14$ at Uppsala University). Frozen tissue sections ( $12 \mu$ m thick; $n=3$ / animal) were cut in a cryostat microtome (Leica CM 1520, Leica Biosystems, Nussloch, Germany) at $-18{ }^{\circ} \mathrm{C}$, and collected on specialcoated, conducting glass slides (indium tin-oxide (ITO), Bruker Daltonics, Bremen, Germany) and stored at $-80^{\circ} \mathrm{C}$.

MALDI Matrix Application. Matrix deposition was carried out using a vacuum sublimation apparatus (Sigma-Aldrich) comprising an inner flat top and an outer bottom attached to each other by an Oring-sealed flange. The chamber was connected to a rough pump attached to a digital vacuum gauge controller and placed in a heated sand bath $\left(\mathrm{SiO}_{2}, 50-70\right.$ mesh particle size, Sigma-Aldrich) on a hot plate (C-MAG HP 4, IKA Werke GmbH \& Co. KG, Staufen, Germany). Sublimation was performed by the following steps. First, ITO- glass slides with the thaw mounted mouse brain tissues were attached to the flat top of the chamber using double-sided conductive copper tape. Then, $300 \mathrm{mg}$ of 1,5-DAN matrix powder was spread evenly on the outer bottom of the sublimation chamber, which was then attached to the top using the O-ring seal. A vacuum of $0.8 \mathrm{mbar}$ was provided by a membrane pump and the cooler was filled with ice $\left(\geq 0{ }^{\circ} \mathrm{C}\right)$ for condensation of the matrix on the sample slides. Sublimation was performed monitoring temperature, time of 
application, and deposited amount of matrix. Here, the amount of deposited matrix onto the tissue sections was optimized varying between $50 \mu \mathrm{g} / \mathrm{cm}^{2}$ and $300 \mu \mathrm{g} / \mathrm{cm}^{2}$. Low amounts yielded too few lipid signals, whereas high amounts yielded too much matrix derived signals. The optimized amount of deposited 1,5-DAN matrix was 120 $\mu \mathrm{g} / \mathrm{cm}^{2}$, which is in a good agreement with previous optimization results. ${ }^{30}$ and was provided by the following sublimation conditions: $20 \mathrm{~min}$ at $130{ }^{\circ} \mathrm{C}$ under a stable vacuum pressure of $0.8 \mathrm{mbar}$.

MALDI Mass Spectrometry. MALDI imaging data were acquired in duplicates from three biological replicates $(n=3)$. Imaging MS analysis of tissue sections were performed on a MALDI TOF/TOF UltrafleXtreme mass spectrometer equipped with SmartBeam II Nd:YAG/355 nm laser operating at $1 \mathrm{kHz}$ (Bruker Daltonics). Profiling and imaging data acquisitions were performed in reflector negative ion mode under optimized delayed extraction conditions in a mass range of 300-3000 Da with a source accelerating voltage of -20 $\mathrm{kV}$. A number of 20 laser shots per pixel were acquired to avoid matrix cluster formation (Figure S-6) and allow for fast data acquisition to avoid matrix sublimation in the ion source. The spatial resolution was $30 \mu \mathrm{m}$ for all biological and technical replicates and $10 \mu \mathrm{m}$ for the colocalization validation experiments. The laser focus was set to small for the $30 \mu \mathrm{m}$ experiments and to minimum for the $10 \mu \mathrm{m}$ acquisition. The mass resolution and accuracy in the lipid mass range (at ca. 800 Da) was of $M / \Delta M 20000$, i.e., $50 \mathrm{ppm}$. External calibration was carried out using peptide calibration standard I (Bruker Daltonics). Image data were reconstructed, root-mean-square (RMS) normalized, and visualized using the Flex Imaging v3.0 software (Bruker Daltonics).

Lipid identification was performed by comparing accurate mass measurements with LIPID MAPS database (www.lipidmaps.org). For confirmation of chemical structures of the selected lipids accumulated on $\mathrm{A} \beta$ plaques, MALDI-LIFT (MS/MS) was performed directly on the plaque deposits in LIFT negative mode. Here, the precursor ion selector was set to $0.4 \%$ of the precursor $\mathrm{m} / z$ for acquisition of parent ions in PARENT mode (600 shots) followed by one point calibration. In FRAGMENT mode, the laser energy was increased by $30 \%$ on the attenuator (global offset 20\%, range 20\%) for post source decay and TOF/TOF characterization (LIFT) of product ions. Post LIFT mother ion suppression (PLMS) was enabled. Fragment spectra were collected until a satisfactory number of productions was observed. Lipid identification was performed based on comparison with diagnostic fragment ions as previously reported ${ }^{39,40}$ or curated in LIPID MAPS.

Data Processing. Prior to analysis all spectra were calibrated externally using the batch-processing function in Flex Analysis (v 3.0, Bruker Daltonics). Calibration spectra were obtained from calibrant solution spots (Protein Calibration Mix 1, Bruker Daltonics) that were placed adjacent to the tissue slides. Image segmentation of the IMS data was evaluated in SciLS (v2014, SciLS GmbH, Bremen, Germany). Regions of interest (ROI) were identified by bisecting k-mean clustering based image segmentation. The ROIs were correlated to mass to charge $(\mathrm{m} / z)$ values using the corresponding function implemented in the software. Average spectra of the annotated ROIs, as well as control ROIs of similar size adjacent to the plaques, were exported as csv files in FlexImaging. Data were reduced through binning. All ROI data were imported into Origin (v. 8.1 OriginLab, Northhampton, MA) and peaks and peak widths were detected on average spectra of each ROI using the implemented peak analyzer function. The determined bin borders for peak integration were exported as tab delimited text file. The bin borders were used for area under curve (AUC) peak integration within each bin (peak-bin) of all individual ROI average spectra using an in-house developed $\mathrm{R}$ script.

Statistical Analysis. Peak area values for all ROI were evaluated using the "Statistical Analysis of Microarray data" (SAM, v.3.0) in Excel (v.2010). The SAM tool, originally developed for microarray analysis, allows comprehensive and unbiased analysis of significant differences in abundance levels between two groups. Two classes, paired analysis of data from plaque ROIs and adjacent control areas was performed for the different anatomical regions, including hippocampus (Hipp) and cortex (Ctx). Further assessment of significant differences in individual lipid signals between the groups was performed with two-tailed, paired $t$ test ( $95 \%$ significance level).

Fluorescent Amyloid Staining and Immunohistochemistry. After MALDI analysis, sections were rinsed in absolute EtOH for 120 $\mathrm{s}$, fixed in $95 \% \mathrm{EtOH} / 5 \% \mathrm{AcOH}$ at $-20{ }^{\circ} \mathrm{C}$ for $8 \mathrm{~min}, 70 \% \mathrm{EtOH}$ at $-20{ }^{\circ} \mathrm{C}$ for $30 \mathrm{~s}, 70 \% \mathrm{EtOH}$ at RT for $30 \mathrm{~s}$, and stored in PBS prior to staining. For amyloid staining, $30 \mathrm{~min}$ incubation in heptameric formyl thiophene acetic acid (h-FTAA), diluted to a final concentration of 3 $\mathrm{mM}$ in PBS, was used. For antibody-based visualization of $\mathrm{A} \beta$, a monoclonal antibody specific for the $\mathrm{A} / 1-16$ epitope $(6 \mathrm{E} 10,1 \mathrm{mg} /$ $\mathrm{mL}$, BioLegend, San Diego, CA) was used as primary antibody, and goat antimouse IgG conjugated to Alexa Fluor 647 (Thermo Fisher Scientific) was used as secondary antibody.

Tissue was blocked for $1 \mathrm{~h}$ at room temperature (RT) in PBS based blocking solution containing 5\% normal goat serum (NGS, Invitrogen, Thermo Fisher Scientific, Carlsbad, CA), 2\% bovine serum albumin (BSA, Sigma-Aldrich), and 0.3\% Triton-X100 (TX100, SigmaAldrich). Incubation with primary antibody (1:500) was performed overnight at $4{ }^{\circ} \mathrm{C}$, and with secondary antibody $(1: 1000)$ for $1 \mathrm{~h}$ at RT, both diluted in PBS based diluent solution (0,05\% NGS, $0.02 \%$ BSA, $0.3 \%$ TX100). Unspecific binding of the secondary antibody was assessed by incubation of in diluent solution without the primary antibody. Each incubation step was followed by $3 \times 5$ min rinse in PBS. Prior to imaging tissue was mounted with Prolong Gold antifade reagent (Thermo Fisher Scientific) and dried for $2 \mathrm{~h}$ at RT. Imaging was performed using a wide field microscope (Axio Observer Z1, Zeiss, Jena, Germany).

\section{ASSOCIATED CONTENT}

\section{S Supporting Information}

The Supporting Information is available free of charge on the ACS Publications website at DOI: 10.1021/acschemneuro.6b00391.

Correlation of immunohistochemical fluorescence stainings of identified plaques with IMS lipid data; single ion images for all biological replicates; MS and MS/MS spectra (PDF)

\section{AUTHOR INFORMATION}

\section{Corresponding Author}

* Mailing address: Department of Psychiatry and Neurochemistry, Sahlgrenska Academy at the University of Gothenburg, Mölndal Hospital, House V, Biskopsbogatan 27, SE-43180 Mölndal, Sweden. E-mail: jorg.hanrieder@neuro.gu. se. Telephone: +46-31-343 2377.

\section{ORCID ${ }^{\circ}$}

Jörg Hanrieder: 0000-0001-6059-198X

\section{Author Contributions}

${ }^{\nabla}$ I.K., D.B., W.M. and J.H. conceived and designed the study. I.K., D.B., W.M., S.S., D.S. and J.H. performed the experiments. I.K, D.B., W.M., S.S., D.S., H.Z., K.B. an J.H. analyzed and discussed the data and wrote the manuscript. I.K. and D.B. contributed equally.

\section{Funding}

The Swedish Research Council VR (\#2014-6447, J.H.; \#20121593, S.S.; \#2013-2546, H.Z.; \#2013-14002, K.B.), the European Research Council (\#681712, H.Z.), Marie Sklodowska Curie Actions (Cofund, Project INCA 600398, J.H.), the Royal Society of Arts and Sciences in Gothenburg (KVVS, J.H.), Alzheimerfonden (J.H., K.B., S.S., D.S.), Demensfonden (J.H.), Hjärnfonden (K.B., S.S.), Jeanssons Stiftelsen (J.H.), Ahlén Stiftelsen (J.H., S.S., D.S.), Svenska Lundbeck-stiftelsen (D.S.), Stiftelsen Gamla Tjänarinnor (J.H., D.S., K.B.), Stohnes Stiftelse (J.H., S.S., D.S.), and Stiftelsen Wilhelm och Martina 
Lundgrens Vetenskapsfond (J.H.) are acknowledged for financial support.

\section{Notes}

The authors declare no competing financial interest.

\section{ACKNOWLEDGMENTS}

The work was in part performed at the go:IMS Gothenburg Imaging MS platform (www.go-ims.gu.se).

\section{ABREVIATIONS}

$\mathrm{AD}$, Alzheimer's disease; $\mathrm{A} \beta$, amyloid beta; $\mathrm{APP}$, amyloid-beta precursor protein; Ctx, cortex; Cer, ceramides; FA, formic acid; h-FTAA, heptameric formyl thiophene acetic acids; GM, gangliosides; Hipp, hippocampus; IHC, immunohistochemistry; IMS, imaging mass spectrometry; ITO, indium tin-oxide; $m / z$, mass-to-charge ratio; MALDI, matrix-assisted laser desorption/ionization; $\mathrm{MeOH}$, methanol; OCT, optimal cutting temperature; ROI, region of interest; RT, room temperature; RMS, root-mean-square; SAM, Statistical Analysis of Microarray data; ST, sulfatides; tgArcSwe, transgenic Arctic and Swedish mutations; TOF, time-of-flight

\section{REFERENCES}

(1) Alonso, A. d. C., Grundke-Iqbal, I., and Iqbal, K. (1996) Alzheimer's disease hyperphosphorylated tau sequesters normal tau into tangles of filaments and disassembles microtubules. Nat. Med. 2, 783-787.

(2) Blennow, K., de Leon, M. J., and Zetterberg, H. (2006) Alzheimer's disease. Lancet 368, 387-403.

(3) Hardy, J. A., and Higgins, G. A. (1992) Alzheimer's disease: the amyloid cascade hypothesis. Science 256, 184.

(4) Yadav, R. S., and Tiwari, N. K. (2014) Lipid integration in neurodegeneration: an overview of Alzheimer's disease. Mol. Neurobiol. 50, $168-176$.

(5) Hirsch-Reinshagen, V., Burgess, B. L., and Wellington, C. L. (2009) Why lipids are important for Alzheimer disease? Mol. Cell. Biochem. 326, 121-129.

(6) Saunders, A. M., Strittmatter, W. J., Schmechel, D., St. GeorgeHyslop, P. H., Pericak-Vance, M. A., Joo, S., Rosi, B., Gusella, J., Crapper-MacLachlan, D., Alberts, M., et al. (1993) Association of apolipoprotein $\mathrm{E}$ allele $\epsilon 4$ with late-onset familial and sporadic Alzheimer's disease. Neurology 43, 1467-1467.

(7) Cutler, R. G., Kelly, J., Storie, K., Pedersen, W. A., Tammara, A., Hatanpaa, K., Troncoso, J. C., and Mattson, M. P. (2004) Involvement of oxidative stress-induced abnormalities in ceramide and cholesterol metabolism in brain aging and Alzheimer's disease. Proc. Natl. Acad. Sci. U. S. A. 101, 2070-2075.

(8) Barrett, P. J., Song, Y., Van Horn, W. D., Hustedt, E. J., Schafer, J. M., Hadziselimovic, A., Beel, A. J., and Sanders, C. R. (2012) The amyloid precursor protein has a flexible transmembrane domain and binds cholesterol. Science (Washington, DC, U. S.) 336, 1168-1171.

(9) Di Paolo, G., and Kim, T. W. (2011) Linking lipids to Alzheimer's disease: cholesterol and beyond. Nat. Rev. Neurosci. 12, 284-296.

(10) Mutoh, T., Hirabayashi, Y., Mihara, T., Ueda, M., Koga, H., Ueda, A., Kokura, T., and Yamamoto, H. (2006) Role of glycosphingolipids and therapeutic perspectives on Alzheimer's disease. CNS Neurol. Disord.: Drug Targets 5, 375-380.

(11) Haughey, N. J., Bandaru, V. V., Bae, M., and Mattson, M. P. (2010) Roles for dysfunctional sphingolipid metabolism in Alzheimer's disease neuropathogenesis. Biochim. Biophys. Acta, Mol. Cell Biol. Lipids 1801, 878-886.

(12) Puglielli, L., Tanzi, R. E., and Kovacs, D. M. (2003) Alzheimer's disease: the cholesterol connection. Nat. Neurosci. 6, 345-351.
(13) Wells, K., Farooqui, A. A., Liss, L., and Horrocks, L. A. (1995) Neural membrane phospholipids in Alzheimer disease. Neurochem. Res. 20, 1329-1333.

(14) Han, X., Holtzman, D. M., McKeel, D. W., Jr., Kelley, J., and Morris, J. C. (2002) Substantial sulfatide deficiency and ceramide elevation in very early Alzheimer's disease: potential role in disease pathogenesis. J. Neurochem. 82, 809-818.

(15) Han, X. (2007) Potential mechanisms contributing to sulfatide depletion at the earliest clinically recognizable stage of Alzheimer's disease: a tale of shotgun lipidomics. J. Neurochem. 103, 171-179.

(16) McLaurin, J., Franklin, T., Fraser, P. E., and Chakrabartty, A. (1998) Structural transitions associated with the interaction of Alzheimer $\beta$-amyloid peptides with gangliosides. J. Biol. Chem. 273, $4506-4515$.

(17) Yanagisawa, K. (2007) Role of gangliosides in Alzheimer's disease. Biochim. Biophys. Acta, Biomembr. 1768, 1943-1951.

(18) Blennow, K., Davidsson, P., Wallin, A., Fredman, P., Gottfries, C.-G., Karlsson, I., Månsson, J.-E., and Svennerholm, L. (1991) Gangliosides in cerebrospinal fluid in'probable Alzheimer's disease'. Arch. Neurol. 48, 1032-1035.

(19) Pernber, Z., Blennow, K., Bogdanovic, N., Månsson, J.-E., and Blomqvist, M. (2012) Altered distribution of the gangliosides GM1 and GM2 in Alzheimer's disease. Dementia Geriatr. Cognit. Disord. 33, $174-188$.

(20) Schaeffer, E. L., Figueiro, M., and Gattaz, W. F. (2011) Insights into Alzheimer disease pathogenesis from studies in transgenic animal models. Clinics 66, 45-54.

(21) Philipson, O., Lord, A., Gumucio, A., O'Callaghan, P., Lannfelt, L., and Nilsson, L. N. (2010) Animal models of amyloid- $\beta$-related pathologies in Alzheimer's disease. FEBS J. 277, 1389-1409.

(22) Lord, A., Kalimo, H., Eckman, C., Zhang, X.-Q., Lannfelt, L., and Nilsson, L. N. G. (2006) The Arctic Alzheimer mutation facilitates early intraneuronal $\mathrm{A} \beta$ aggregation and senile plaque formation in transgenic mice. Neurobiol. Aging 27, 67-77.

(23) McDonnell, L. A., and Heeren, R. M. A. (2007) Imaging mass spectrometry. Mass Spectrom. Rev. 26, 606-643.

(24) Chaurand, P., Cornett, D. S., Angel, P. M., and Caprioli, R. M. (2011) From whole-body sections down to cellular level, multiscale imaging of phospholipids by MALDI mass spectrometry. Mol. Cell. Proteomics 10, O110. 004259.

(25) Sugiura, Y., Shimma, S., Konishi, Y., Yamada, M. K., and Setou, M. (2008) Imaging mass spectrometry technology and application on ganglioside study; visualization of age-dependent accumulation of C20-ganglioside molecular species in the mouse hippocampus. PLoS One 3, e3232.

(26) Caprioli, R. M., Farmer, T. B., and Gile, J. (1997) Molecular imaging of biological samples: localization of peptides and proteins using MALDI-TOF MS. Anal. Chem. 69, 4751-4760.

(27) Hanrieder, J., Ljungdahl, A., Fälth, M., Mammo, S. E., Bergquist, J., and Andersson, M. (2011) L-DOPA-induced dyskinesia is associated with regional increase of striatal dynorphin peptides as elucidated by imaging mass spectrometry. Mol. Cell. Proteomics 10, M111. 009308.

(28) Carlred, L., Michno, W., Kaya, I., Sjovall, P., Syvanen, S., and Hanrieder, J. (2016) Probing amyloid-beta pathology in transgenic Alzheimer's disease (tgArcSwe) mice using MALDI imaging mass spectrometry. J. Neurochem. 138, 469-478.

(29) Hankin, J. A., Barkley, R. M., and Murphy, R. C. (2007) Sublimation as a method of matrix application for mass spectrometric imaging. J. Am. Soc. Mass Spectrom. 18, 1646-1652.

(30) Thomas, A. l., Charbonneau, J. L., Fournaise, E., and Chaurand, P. (2012) Sublimation of new matrix candidates for high spatial resolution imaging mass spectrometry of lipids: enhanced information in both positive and negative polarities after 1, 5-diaminonapthalene deposition. Anal. Chem. 84, 2048-2054.

(31) Nilsson, K. P., Aslund, A., Berg, I., Nystrom, S., Konradsson, P., Herland, A., Inganas, O., Stabo-Eeg, F., Lindgren, M., Westermark, G. T., Lannfelt, L., Nilsson, L. N., and Hammarstrom, P. (2007) Imaging 
distinct conformational states of amyloid-beta fibrils in Alzheimer's disease using novel luminescent probes. ACS Chem. Biol. 2, 553-560. (32) He, X., Huang, Y., Li, B., Gong, C. X., and Schuchman, E. H. (2010) Deregulation of sphingolipid metabolism in Alzheimer's disease. Neurobiol. Aging 31, 398-408.

(33) Ariga, T., Wakade, C., and Yu, R. K. (2011) The pathological roles of ganglioside metabolism in Alzheimer's disease: effects of gangliosides on neurogenesis. Int. J. Alzheimer's Dis. 2011, 1.

(34) Ariga, T., Kobayashi, K., Hasegawa, A., Kiso, M., Ishida, H., and Miyatake, T. (2001) Characterization of high-affinity binding between gangliosides and amyloid $\beta$-protein. Arch. Biochem. Biophys. 388, 225230.

(35) Yanagisawa, K., Odaka, A., Suzuki, N., and Ihara, Y. (1995) GM1 ganglioside-bound amyloid beta-protein (A beta): a possible form of preamyloid in Alzheimer's disease. Nat. Med. 1, 1062-1066.

(36) Kakio, A., Nishimoto, S.-i., Yanagisawa, K., Kozutsumi, Y., and Matsuzaki, K. (2001) Cholesterol-dependent formation of GM1 ganglioside-bound amyloid $\beta$-protein, an endogenous seed for Alzheimer amyloid. J. Biol. Chem. 276, 24985-24990.

(37) Han, X. L., Holtzman, D. M., McKeel, D. W., Kelley, J., and Morris, J. C. (2002) Substantial sulfatide deficiency and ceramide elevation in very early Alzheimer's disease: potential role in disease pathogenesis. J. Neurochem. 82, 809-818.

(38) Katsel, P., Li, C., and Haroutunian, V. (2007) Gene expression alterations in the sphingolipid metabolism pathways during progression of dementia and Alzheimer's disease: a shift toward ceramide accumulation at the earliest recognizable stages of Alzheimer's disease? Neurochem. Res. 32, 845-856.

(39) Woods, A. S., Colsch, B., Jackson, S. N., Post, J., Baldwin, K., Roux, A., Hoffer, B., Cox, B. M., Hoffer, M., Rubovitch, V., et al. (2013) Gangliosides and ceramides change in a mouse model of blast induced traumatic brain injury. ACS Chem. Neurosci. 4, 594-600.

(40) Hsu, F.-F., Turk, J., Stewart, M. E., and Downing, D. T. (2002) Structural studies on ceramides as lithiated adducts by low energy collisional-activated dissociation tandem mass spectrometry with electrospray ionization. J. Am. Soc. Mass Spectrom. 13, 680-695.

(41) Kracun, I., Kalanj, S., Talan-Hranilovic, J., and Cosovic, C. (1992) Cortical distribution of gangliosides in Alzheimer's disease. Neurochem. Int. 20, 433-438.

(42) Kracun, I., Rosner, H., Drnovsek, V., Heffer-Lauc, M., and Lauc, G. (2002) Human brain gangliosides in development, aging and disease. Int. J. Dev. Biol. 35, 289-295.

(43) Svennerholm, L., and Gottfries, C. G. (1994) Membrane Lipids, Selectively Diminished in Alzheimer Brains, Suggest Synapse Loss as a Primary Event in Early-Onset Form (Type I) and Demyelination in Late-Onset Form (Type II). J. Neurochem. 62, 1039-1047.

(44) Kolter, T., and Sandhoff, K. (2010) Lysosomal degradation of membrane lipids. FEBS Lett. 584, 1700-1712.

(45) Dufresne, M., Guneysu, D., Patterson, N. H., Marcinkiewicz, M. M., Regina, A., Demeule, M., and Chaurand, P. (2016) Multimodal detection of GM2 and GM3 lipid species in the brain of mucopolysaccharidosis type II mouse by serial imaging mass spectrometry and immunohistochemistry. Anal. Bioanal. Chem., DOI: $10.1007 /$ s00216-016-0076-x. 